ove $\varepsilon^{\prime}$ ’ $j$ l complemento del modulo $\varepsilon$, e si avrebbe

$$
\varepsilon^{\prime 2}=\frac{k k_{1}+2 k z}{k k_{1}+2 k_{\mathrm{x}} z}, \operatorname{sen}^{2} \theta=\frac{k+2 z}{k_{1}+2 z}
$$

Di più per le formole date da Lcgendre la funzione $\Pi(n, \varepsilon)$ si potrà csprimere per le funioni incomplete, c complete di prima, c seconda specie, e che tralasciamo di escguire. Osserveremo in ultimo, che per il paraboloide di rivoluzione attorno l'asse delle z si avrelobe,

d'onde

$$
k=k_{1}, \quad n=0, \quad \varepsilon=0 .
$$

c quindi per la superficie

$$
\mathrm{F}(\varepsilon)=\mathrm{E}(\varepsilon)=\mathrm{I}(n, \varepsilon) \doteq{ }_{2}^{\pi}
$$

$$
\mathrm{S}=\frac{\pi}{2.3}\left(\sqrt{k(k+2 z)^{3}}-k^{2}\right)
$$

valore di gì̀ noto, e facile ad esser verificato da una diretta integrazione.

Roma 3. Novembre 1862.

\title{
PUBLICAZIONI RECENTI
}

Lighenti - Ricerche geometriche, ed idrometriche per la scuola degli Ingegneri di Roma $2^{2}$ Ediz. con nuove note el . . Pisa 1862.

Schiaparel LI. G. V. - Sulla trasformazione geometrica delle figure ed in particolare sulla trasformazione Iperbolica in $4^{\circ}$ 'T'orino 1862.

CRemona L. - Introduzione ad una teoria geometrica delle curve piane, in $\AA^{\circ}$. Bologna 1862.

Mèsarfa L. F. - Note sur l'Effet du choc de l'eau dans les conduites, in $4^{\circ}$. Turin, 1862.

Lacroix, - Traité élémentaire de calcul diff. et de calcul Int. 6. ${ }^{c}$ Edit. 2 vol. in $\mathbf{s}^{\text {. }}$ avec de notes par M. M. Hermite et Serret.

SALMon, G. - A Treatise on the analitic geometry of three dimensions vol. in 8 : Dublin, 1862.

Ifermite $C_{\text {H. }}$ - Sur la théorie des fonctions elliptiques, et ses applications à l'arithmetique, in 4: Paris, 1862.

Alégret - Essai sur le calcul des quaternions de M. W. Hamilton Paris, 1862.

Desboves. - Théorie nouvelle des normales aux surfaces du second ordre. Paris, 1862.

Lamarle - Exposé géometrique du calcul differentiel et intégral précedé de la cinématique du point, de la droite et du plan. Bruxelles, 1861.

Willert A. T. - Disquisitiones de curvaturis nonnullarum secundi ordinis superficierum, in $4^{\circ}$. Greifswald, 1862. 